\title{
A CHALLENGE TO TERABIT PERPENDICULAR SPINIC STORAGE
}

\author{
Yoshihisa NAKAMURA \\ Research Institute of Electrical Communication, Tohoku University \\ 2-1-1, Katahira, Aoba-ku, Sendai, Miyagi 980-77, Japan
}

\begin{abstract}
Magnetic data storage possesses the potential for extremely high bit density exceeding $1 \mathrm{~Tb} / \mathrm{cm}^{2}$ or about $10 \mathrm{~Tb} /$ inch ${ }^{2}$, which corresponds to storing each bit into each ferromagnetic fine particle composing a magnetic storage medium. We call this 'perpendicular spinic storage (PSS)' because this tremendous potential of extremely high bit density could only be established by perpendicularly magnetizing ferromagnetic particles, or parallel spin clusters, arranged systematically and oriented perpendicularly on a substrate. The possibility and the background of terabit PSS in magnetic storage technology are discussed from the viewpoint of theoretical considerations and the current situation in high density recording. To approach this tremendous possibility, main-pole thick width (MPTW) recording of several hundreds nanometer in width and dispersive writing and collective reading (DWCR) with a multipletrack head are proposed.
\end{abstract}

\section{Introduction}

Recently, words such as super information highways, multimedia, video-on-demands and interactive communications have become popular in the new information communication society. To give full play to their expected performance, information storage systems as well as communication and computer systems are required.

Information storage technology plays a very important role not only in storing a huge quantity of information including sounds and images but also in transporting and distributing it through time as well as space. Hence, major progress in high storage density is always and fatally required.

Although magnetic data storage has a long history of about a hundred years, it has still increased the areal bit density at a rate of $\mathbf{1 0}$ times or more every $\mathbf{1 0}$ years during the past 30 years. If this trend continues, the areal bit density is expected to exceed $1 \mathrm{~Tb} / \mathrm{cm}^{2}$ or about $10 \mathrm{~Tb} / \mathrm{inch}^{2}$ in the mid-21th century (Fig. 1). Whether magnetic storage has such a tremendous potential or not is a very interesting subject.

This paper will propose that such a tremendous possibility can be established only by perpendicular magnetizing methods in which each bit is able to be stored into each ferromagnetic fine particle composing a magnetic storage medium. Such a possibility in magnetic information storage

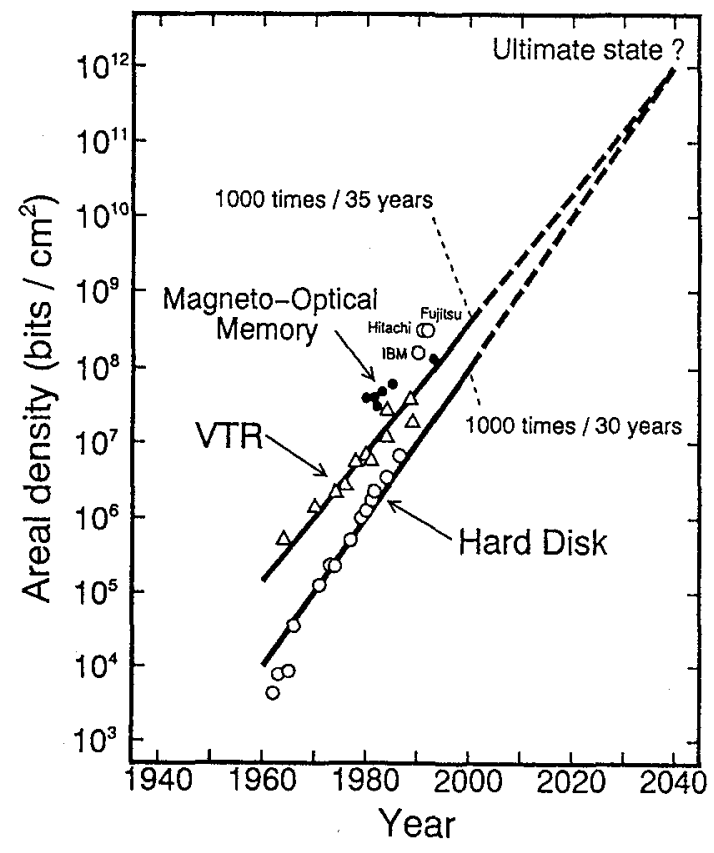

Fig. 1 Trend of high areal bit density in magnetic data storage system. 
technology will be discussed from theoretical considerations and the current situation in high-density recording. Several ways to realize the possibility will then be proposed.

\section{Spinic Storage}

\subsection{Principle}

A magnetic recording medium is fundamentally an assembly of a great number of ferromagnetic single-domain fine particles. The particles are clusters of electron spins arranged in parallel which contributes to the ferromagnetic properties. If a uniaxial magnetic anisotropy is given to the particle by elongating its shape or by using a material having a crystalline anisotropy, a binary data of ' 0 ' or ' 1 ' can be stored using only the positive or negative direction of magnetization or electron spin along the easy magnetization axis of the particle or the spin cluster (Fig. 2).

We can not call it 'spin memory' because each bit is not stored by the direction of each electron spin. However, we can call this 'spin caluster memory' or 'spinic memory'. If a storage medium is made of tremendous numbers of fine magnetic particles having the same shape and size which

0
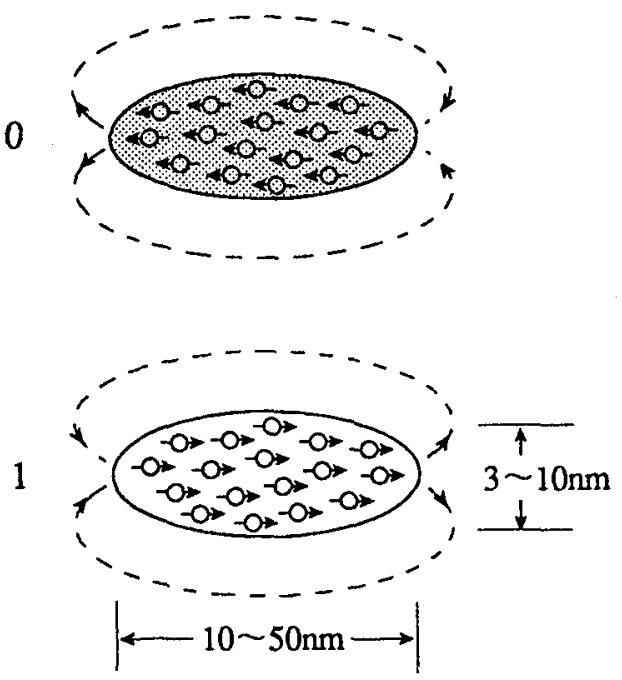

Fig. 2 Principle of spinic memory: A bit is stored using the direction of parallel electron spins in a uniaxial anisotropic ferromagnetic single-domain particle. are arranged systematically onto a substrate and a binary digital data row can be successively written by polarity change of magnetization of each particle, we can call this 'spinic data storage'.

\subsection{Longitudinal spinic storage (LSS)}

We suppose that ferromagnetic single-domain elongated fine particles of $L$ in length and $R$ in diameter are systematically arranged and oriented in the longitudinal direction (Fig. 3). When the direction of spins or magnetization in a particle can be changed one by one to positive or negative

(a) Writing and reading in LSS

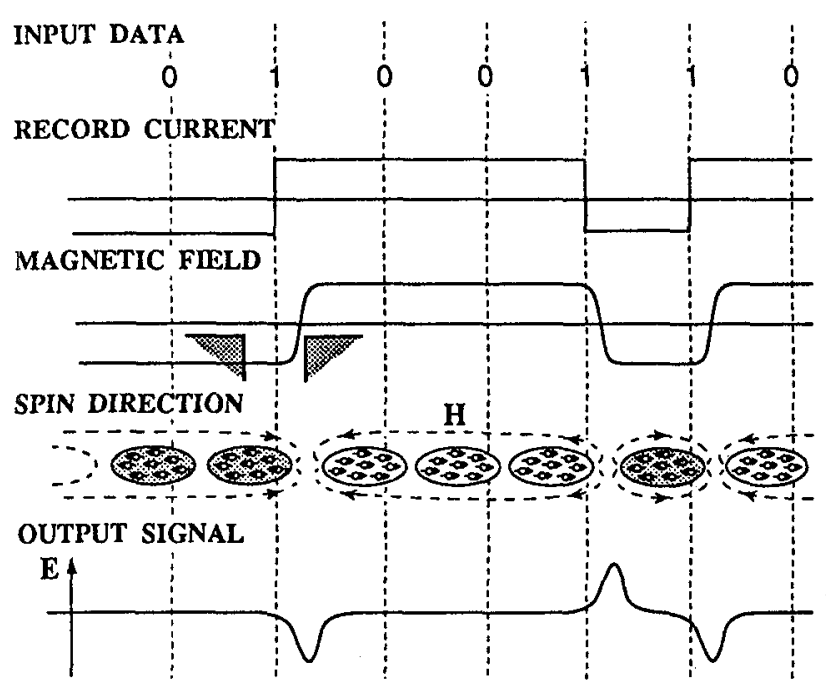

(b) Data storage state on LSS media

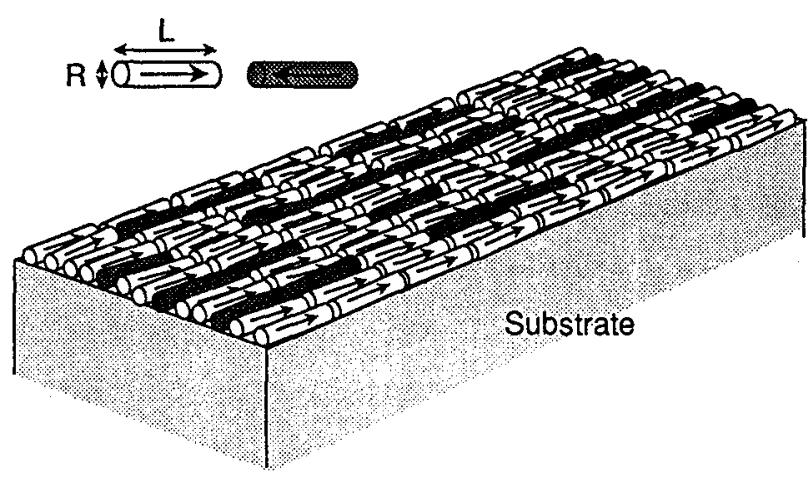

Fig. 3 Principle of longitudinal spinic storage (LSS): (a) Writing and reading principle, and (b) Schematic diagram of data storage state. 
depending on ' 1 ' or ' 0 ' of data, a binary data row can be written at a bit interval of $L$. We call this 'longitudinal spinic storage (LSS)'. Data of ' 1 ' and ' 0 ' are written between elongated magnetic particles arranged in the longitudinal direction and restored by detecting whether the magnetic field between the magnetic particles is generating or not. The magnetic field is generated only between particles which have reversed magnetization directions. This is the ultimate state of the current longitudinal magnetic recording.

The minimum area of a bit cell in LSS can be roughly estimated by the products of $L$ and $R$ in a magnetic particle. This area will be $250 \mathrm{~nm}^{2}$ when $L$ is $50 \mathrm{~nm}$ and $R$ is $5 \mathrm{~nm}$. This means that LSS has the potential of an extremely high density data storage which may reach $0.4 \mathrm{~Tb} / \mathrm{cm}^{2}$. When magnetic particles are a spherical shape having uniaxial magneto-crystalline anisotropy and diameter of about 10 $\mathrm{nm}$ which is the minimum order of diameter to preserve ferromagnetic behavior [1], the bit area can be reduced further to the order of $100 \mathrm{~nm}^{2}$, corresponding to about 1 $\mathrm{Tb} / \mathrm{cm}^{2}$.

In LSS, however, a strong mutual repelling force is applied between the particles where data of ' 1 ' is written. The magnetization directions are thus tilted from the easy to hard magnetization direction or from the longitudinal to perpendicular direction. The magnetization may be made anti-parallel and finally be made to reverse, depending on the circumstances.

To avoid such instability, the magnetic particles should be made as small as possible and should be arranged in only a single layer of particles on a substrate to reduce the repelling force. Furthermore, to resist the repelling force, the magnetic anisotropy of the particles should be made as intense as possible and the easy axis should be arranged as much in a head traveling direction as possible. These conditions correspond to making a magnetic layer of recording media as thin and the coercive force as high as possible in the current high-density magnetic media.

\subsection{Perpendicular spinic storage (PSS)}

If spinic storage media are made so that the easy magnetization axes of particles are perpendicular to the substrate, ' 1 ' or ' 0 ' can be written by making the magnetization direction anti-parallel or parallel (Fig. 4). The data written between the magnetic particles can be read by detecting whether the polarity of the magnetic field is reversed or not. This can be called 'perpendicular spinic storage (PSS)', because this is the ultimate state in perpendicular magnetic recording [2].

Since attractive forces act on each particle magnetized in the anti-parallel direction where data of ' 1 ' is written, the magnetization directions of particles is always perpendicular to the substrate. This implies that the particles are freed from the so called demagnetizing field, or interparticle interaction field generating the repelling force. Although a mutual repelling force acts on adjacent particles magnetized in parallel where ' 0 's are written, the magnetization polarity never reverses because the energy of the perpendicular magnetic anisotropy exceeds that of the repelling force. Therefore a ' 0 ' is never changed to a ' 1 '.

In PSS, a data row can be written stably even at an extremely high density of bit interval $R$ corresponding to the

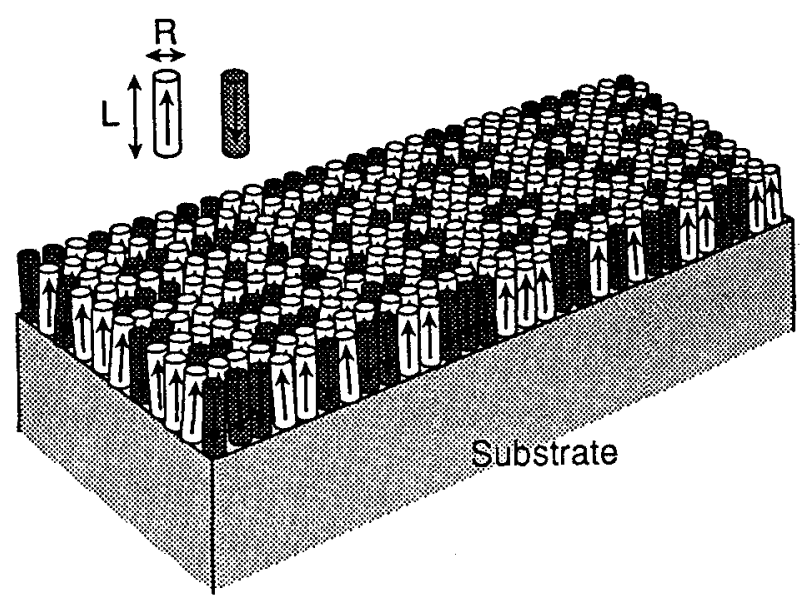

Fig. 4 Schematic diagram of data storage state in perpendicular spinic storage (PSS). 
diameter of an elongated acicular or columnar magnetic particle. If the minimum bit area can be roughly estimated by $R^{2}$, PSS has the potential of $4 \mathrm{~Tb} / \mathrm{cm}^{2}$ or $25 \mathrm{~Tb} / \mathrm{inch}^{2}$ when the length $L$ of a columnar particle is assumed to be $50 \mathrm{~nm}$ and diameter $R, 5 \mathrm{~nm}$.

\section{Background}

\subsection{Computer simulation}

Using a magnetic recording simulator in which media are assumed to be like a spinic storage media $[3,4]$, magnetic flux densities $B_{n}$ on the surface after writing can be computed theoretically for various bit densities assuming various heads, media and writing conditions. Thus the bit density characteristics of an assumed medium can be obtained by plotting the peak values $B_{n \text { peak }}$ of $B_{n}$ against bit densities $D$.

On the curve, the maximum value $B_{n \text { max }}$ of $B_{n \text { peak }}$ at a low bit density and the $50 \%$ bit density $D_{s, 50}$ at which $B_{\text {mpeak }}$ is $50 \%$ of $B_{n, \max }$ correspond respectively to the maximum readout voltage at a lower bit density and the utilizable highest bit density for the medium assumed. Hence, we can theoretically estimate the abilities of various media for high density recording and investigate media parameters correlated with high-density recordings in both LSS and PSS [4].

\subsubsection{Longitudinally oriented media}

In the combination of longitudinally oriented spinic storage media and ring heads, we found the following media parameters correlated with $B_{n, \max }$ and $D_{s, 50}$,

$$
B_{n, \max }=K_{B, \text { lomg }}\left(\delta M, H_{c}\right)^{0.6}
$$

and

$$
D_{s f, s 0}=K_{D, \text { long }}\left(\delta M_{r} / H_{c}\right)^{-0.4}
$$

where $K_{B, \text { long }}$ and $K_{D, \text { long }}$ are constants, $\delta$ is the thickness of a magnetic layer of a storage medium, and $M_{r}$ and $H_{c}$ are the remanent magnetization and the coercive force in the direction of the easy axis, which are the average of those of particles composing the medium. The simulation was conducted assuming a head-to-media spacing of $20 \mathrm{~nm}$. A larger exponent in the equations indicates a closer correlation. From the product of equations (1) and (2), or

$$
\left(B_{n, \max } D_{s f, 50}\right)=K_{B, \text { long }} K_{D, \text { long }}\left(\delta M_{r}\right)^{0.2} H_{c}
$$

we can see that $H_{c}$ contributes more than the product of $M_{r}$ and $\delta$ to both a higher readout voltage and a utilizable recording density. This implies that the demagnetizing field or the magneto-static interaction between the particles still affects high-density recording even in much thinner LSS media.

\subsubsection{Perpendicularly oriented media}

When a single-pole head is combined with a doublelayered media composed of a perpendicularly oriented data storage layer and a soft magnetic underlayer $[2,4], B_{n, \max }$ and $D_{s ; 50}$ are closely correlated with media parameters as follows,

$$
B_{n, \max }=K_{B, p e r p}\left(\delta H_{c}^{2} / \sigma_{h c}\right)^{0.55}
$$

and

$$
D_{s, 50}=K_{D, p e r p}\left(\delta \sigma_{h c} / M_{s}\right)^{-0.55}
$$

where $K_{B, p e r p}$ and $K_{D, p e r p}$ are constants, $\delta$ is the thickness of a data storage layer, $H_{c}$ and $M_{s}$ are the coercive force and the saturation magnetization perpendicular to the medium surface (also the average of those of particles), and $\sigma_{h c}$ is the standard deviation in the dispersion of coercive forces $h_{c}$ of particles.

From the product of equations (4) and (5),

$$
\left(B_{n, \max } \cdot D_{s, 50}\right)=K_{B, p e r p} K_{D, p e r p}\left(H_{c} / \sigma_{h c}\right)^{1.1} M_{s}^{0.55}
$$

we can find that a narrower $\sigma_{h c}$ of magnetic particles affects primarily both higher reproduced output and higher data storage density. Coercive force $H_{c}$ of the media contributes to increasing the readout voltage at a lower density but does not improve bit density characteristics. The saturation magnetization $M_{s}$, however, strongly affects higher storage density. 
A narrower $\sigma_{h c}$ of the data storage layer causes higher rectangularity of the $\mathrm{M}-\mathrm{H}$ loops measured in the easy magnetization direction [5]. A larger $M_{s}$ of the storage layer induces stronger magneto-static interaction with a single-pole head, which results in the stronger and sharper perpendicular field distribution of the head [6]. These facts imply that the high-density recording in PSS is determined only by the rectangularity of $\mathrm{M}-\mathrm{H}$ loop of a medium and the sharpness of the magnetic field distribution of a head, because interparticle interaction or demagnetizing fields do not affect the high-density storage in PSS.

A perpendicularly oriented $\mathrm{Co}-\mathrm{Cr}$ alloy film developed for perpendicular magnetic recording consists of Co-rich hcp crystalline fine magnetic columnar particles, growing perpendicularly from the substrate and bounded by a $\mathrm{Cr}$-rich non-magnetic thin layer [7]. The $\sigma_{h c}$, representing the dispersion of particle coercivity $h_{c}$, strongly correlates with

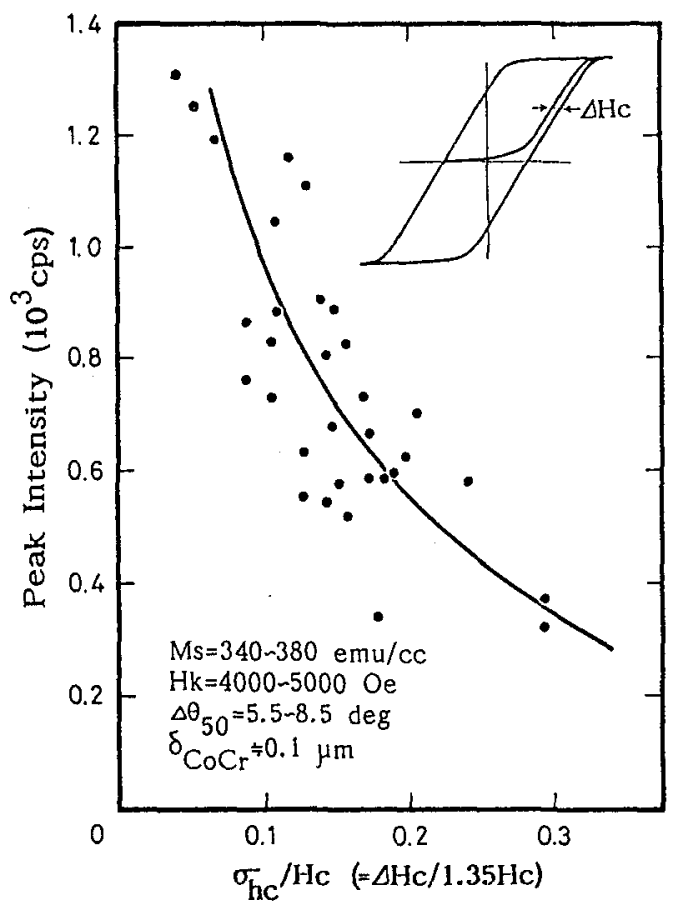

Fig. 5 Correlation of peak intensity of X-ray diffraction from hcp (002) with dispersion $\sigma_{h c}$ of particle coercive forces $h_{c}$ in Co-Cr film: The $\sigma_{h c}$ is estimated by $\Delta H_{c}$ of the hysteresis loop. an intensity of X-ray diffraction from the (002) plane of hcp crystalline particles (Fig. 5) [5]. This means that a higher crystallinity of particles results in a narrower $\sigma_{h c}$ and a higher $\mathrm{M}-\mathrm{H}$ loop rectangularity of the medium.

Consequently, we can conclude that an extremely high areal bit density of several $\mathrm{Tb} / \mathrm{cm}^{2}$ which corresponds to a bit area reaching the cross sectional area of an elongated magnetic crystalline particle will be achieved only by PSS, in which a single-pole head is combined with a perpendicularly oriented particulate data storage layer backed with a soft magnetic underlayer.

\subsection{Recent magnetic media}

Magnetic storage media are fundamentally assemblies of ferromagnetic acicular fine particles of several hundreds nanometer in length and several tens nanometer in diameter. A higher storage density and a lower media noise are produced by particulate magnetic media made of finer particles dispersed uniformly. Ba-ferrite particles developed for high-density recording are much finer hexagonal-platelets of several tens namometer in diameter and several nanometer in thickness [8].

On the other hand, it has been shown that perpendicularly oriented $\mathrm{Co}-\mathrm{Cr}$ columnar particulate films developed for perpendicular magnetic recording exhibit a much lower medium noise independently of bit density [9]. Since then, longitudinally anisotropic $\mathrm{Co}-\mathrm{Cr}-\mathrm{M}$ system alloy thin films were developed for longitudinal magnetic recording with a higher density and a lower noise. They are deposited by sputtering on the tops of non-magnetic $\mathrm{Cr}$ columnar particles of several tens nanometer in diameter growing perpendicularly from the substrate (Fig. 6) [10].

In recent longitudinal magnetic recording, bit area has been gradually decreasing while bit density has been increasing. If a storage medium is an assembly of particles of several tens nanometer in diameter, a bit may be written in several ten-thousands of particles in the current diskdrive of $400 \mathrm{Mb} /$ inch $^{2}$ but it will be written in several 
thousands of particles in the next disk-drives of $2 \mathrm{~Gb} / \mathrm{inch}^{2}$ [11], and moreover, several hundreds of particles in the future ones of $10 \mathrm{~Gb} /$ inch $^{2}$ [12].

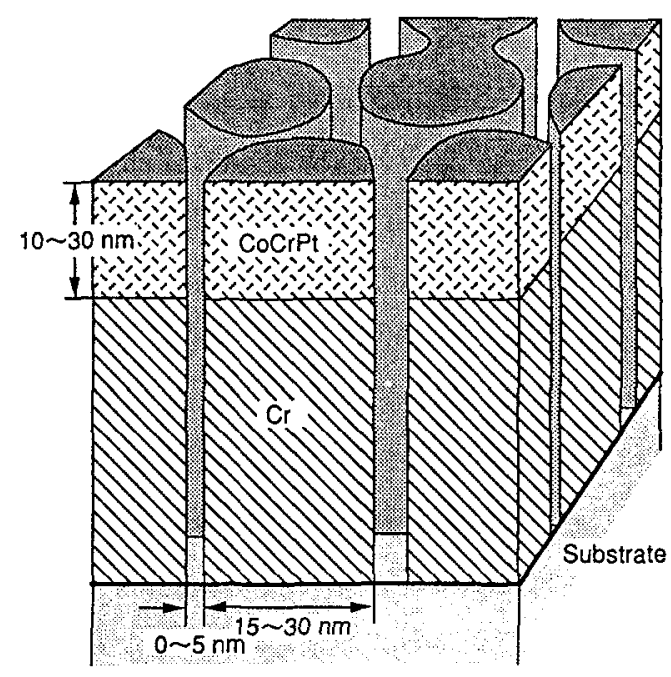

Fig. 6 Schematic diagram of recent longitudinal magnetic recording media: $\mathrm{Co}-\mathrm{Cr}-\mathrm{M}$ particles are deposited on the tops of $\mathrm{Cr}$ columnar particles by sputtering.

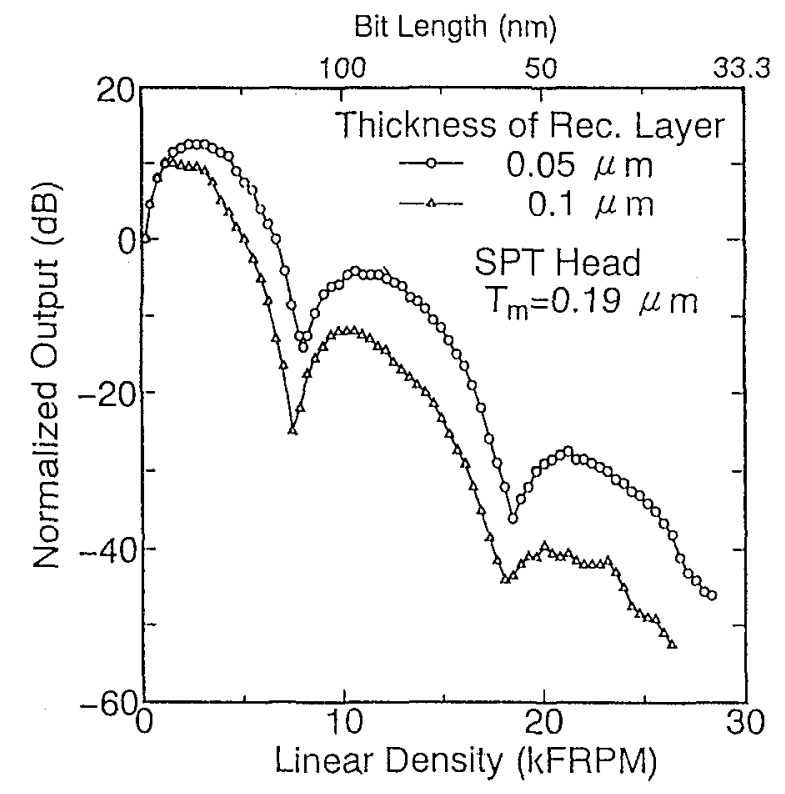

Fig. 7 Bit density characteristics for a perpendicular magnetic media and a single-pole head: Signals of extremely high bit densities (over $25 \mathrm{kFRPM}$ ) can be written and read.
In contrast, we have already confirmed many times by experiments $[4,6,13]$ and also by computer simulations $[4$, 14] that an extremely high bit density recording exceeding $25 \mathrm{kFRPM}$ or $600 \mathrm{kFRPI}$ is achievable through perpendicular magnetic recording. The bit interval of several tens of nanometers corresponds to the diameter of several columns of Co-Cr crystalline particles (Fig. 7). Supposing that the same writing resolution can be realized in the directions of head track width and head traveling, we can expect that a bit will be written in several tens of particles, which corresponds to a bit area of several thousands square-nanometers or an areal bit density of several tens gigabits per square-centimeters.

Although we do not yet have any method for writing each bit into individual magnetic particles and reading these out, we can conclude that magnetic storage technologies will be surely and gradually moving toward the era of spinic data storage.

\section{Approaches}

\subsection{Subjects}

At least, three matters must be solved or developed to realize perpendicular spinic storage (PSS). First, we must develop PSS media made of uniaxial anisotropic fine magnetic single-domain particles with the same shape and size, arranged systematically and oriented perpendicularly to the substrate. Fortunately, in perpendicularly anisotropic $\mathrm{Co}-\mathrm{Cr}$ alloy films deposited by sputtering and so on, a particulate structure of isolated fine columnar crystalline particles was confirmed to grow perpendicularly to the substrate [7]. Although we can not yet systematically arrange magnetic crystalline particles by artificial methods like a sputtering, PSS media should be developed in the near future after the deposition mechanisms of particulate magnetic alloy films have been clarified.

Second, we must develop high resolution sensitive PSS heads so that each bit can be written in each individual 
magnetic particle and can be read it. A single-pole head can be made to have a much stronger and sharper magnetic field around the tip of the main-pole by combining with a perpendicularly anisotropic $\mathrm{Co}-\mathrm{Cr}$ alloy media lined with a soft magnetic underlayer because of the strong magnetic interaction between the main-pole of a head and the double-layered medium [6]. As a result, signals of a bit density exceeding $25 \mathrm{kFRPM}$ with bit interval of less than $50 \mathrm{~nm}$ (diameters of several particles) can be written and read with the single-pole head $[4,6,13]$. To proceed toward spinic writing and reading, therefore, we must put stress developing much narrower track single-pole heads of several hundreds nanometer or less with a much higher $\mathbf{S} / \mathbf{N}$.

Third, we should introduce a novel signal processing scheme. The current track-by-track recording method may face serious difficulties in tracking-servo operation as the track width is reduced. One idea to overcome these difficulties is to write a binary digital signal row dispersively in the track direction on a storage medium with a multiple track head composed of several subheads having an extremely narrow track [15] (Fig. 8). The data written dispersively can be read collectively with a single track head with a wide track, and then they are converted into the original digital data row. We call this Dispersive-Writing and Collective-Reading (DWCR). In this way, we can avoid the difficulty in the servo for extremely narrow tracking.

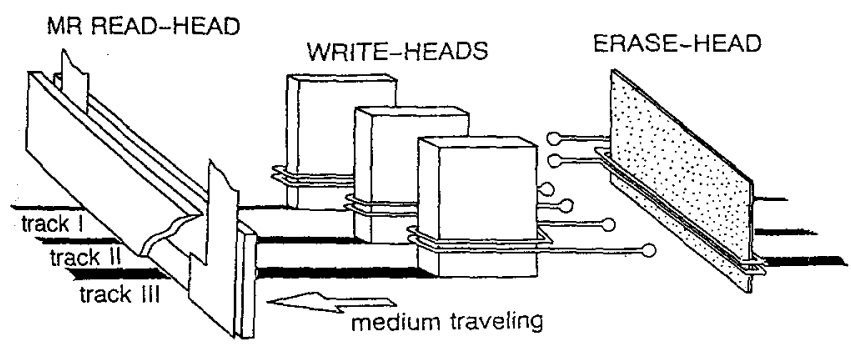

Fig. 8 Method of dispersive writing and collective reading (DWCR): Data are written in parallel by a multiple track head and read collectively by a single track head.

\subsection{Main-pole thick width (MPTW) writing}

Usually a single-pole head travels parallel to the mainpole thickness for writing and reading. Thus the main-pole width $W_{m}$ corresponds to the track width $W_{i}$. To make a track narrow, the main-pole film must be made narrow by etching. However, it is difficult to achieve the width of less than $1 \mu \mathrm{m}$.

The magnetic field distribution at the edges of a mainpole of a single-pole head is as sharp along the direction parallel to the width as it is along the thickness direction (Fig. 9) [15]. We attempted a sub-micron track width
(a) Single-pole head

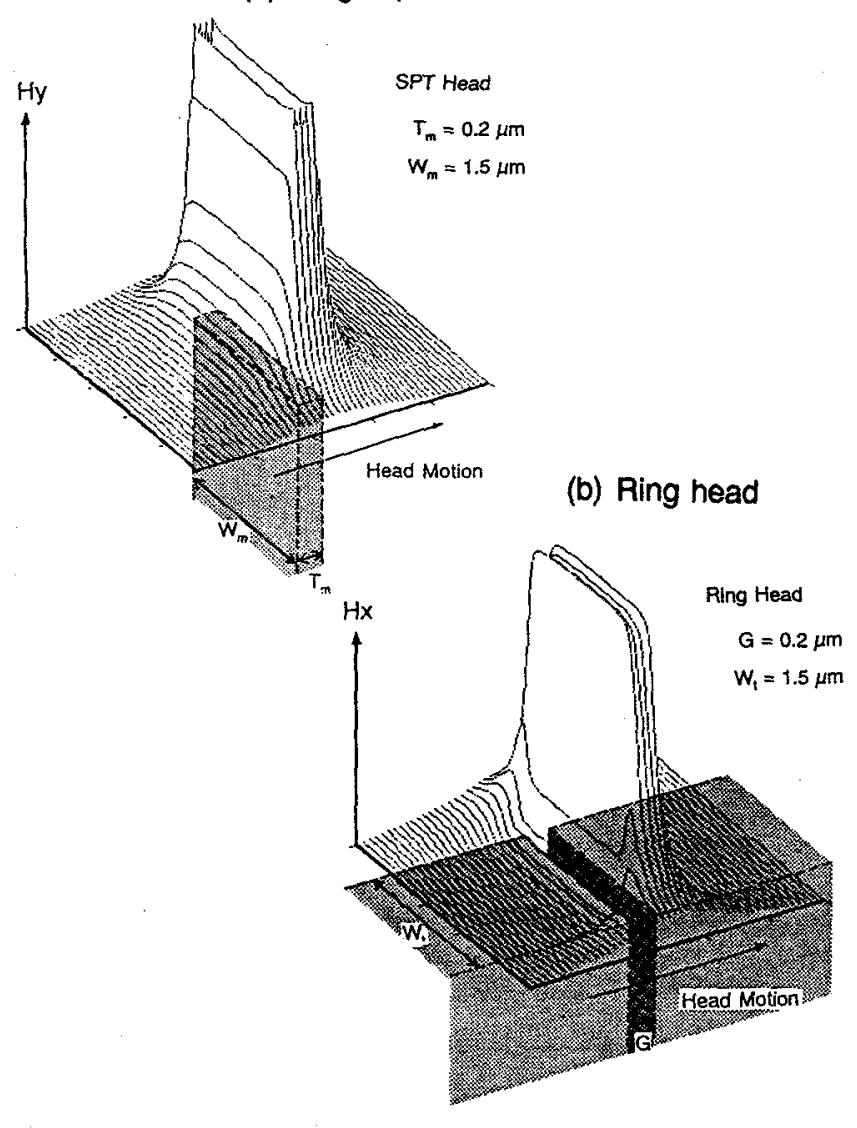

Fig. 9 Three-dimensional magnetic field distribution of (a) single-pole and (b) ring heads: The sharpness of a singlepole head along the main-pole width is the same as that along the thickness direction. 
writing by traveling a single-pole head along the direction parallel to the main-pole width. We call this 'main-pole thick width (MPTW) writing'. The track width $W_{t}$ was reduced to the main-pole thickness $T_{m}$ of several hundreds nanometer, which can easily and accurately be controlled by only the deposition rate and time.

(a) Isolated

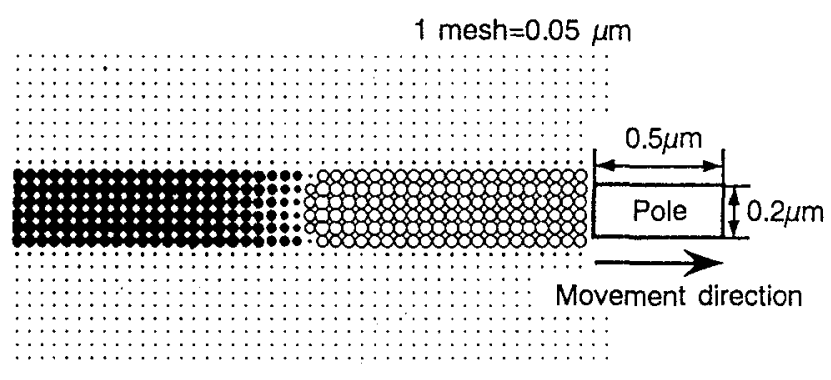

(b) 20 KFRPM

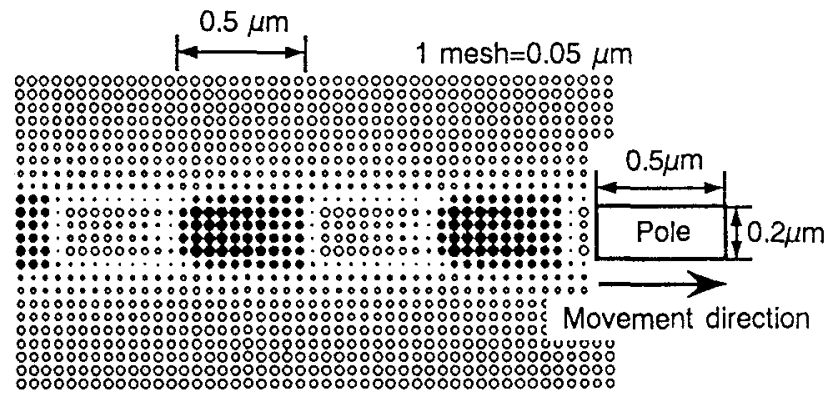

Fig. 10 Magnetization states obtained by three-dimensional computer simulations of main-pole thick width (MPTW) writing: Transitions and track edges are clear even at a track width of $0.2 \mu \mathrm{m}$.

Using a three-dimensional computer simulation on MPTW writing, much narrower tracks (several hundreds nanometer in width) were clearly confirmed without any spreading of a track width or position shift of transitions along the track width direction (Fig. 10) [16]. Furthermore, several experiments on MPTW track writing have been conducted with a single-pole head of $300 \mathrm{~nm}$ in main-pole thickness, and the magnetized states have been observed by magnetic force microscopy (MFM) $[15,16]$ (Fig. 11). Much sharper and narrower tracks of about $400 \mathrm{~nm}$ in width were confirmed even at 8000 FRPM. Bit areas were about $0.05 \mu \mathrm{m}^{2}$ corresponding to about $13 \mathrm{~Gb} /$ inch $^{2}$ in guardbandless writing.

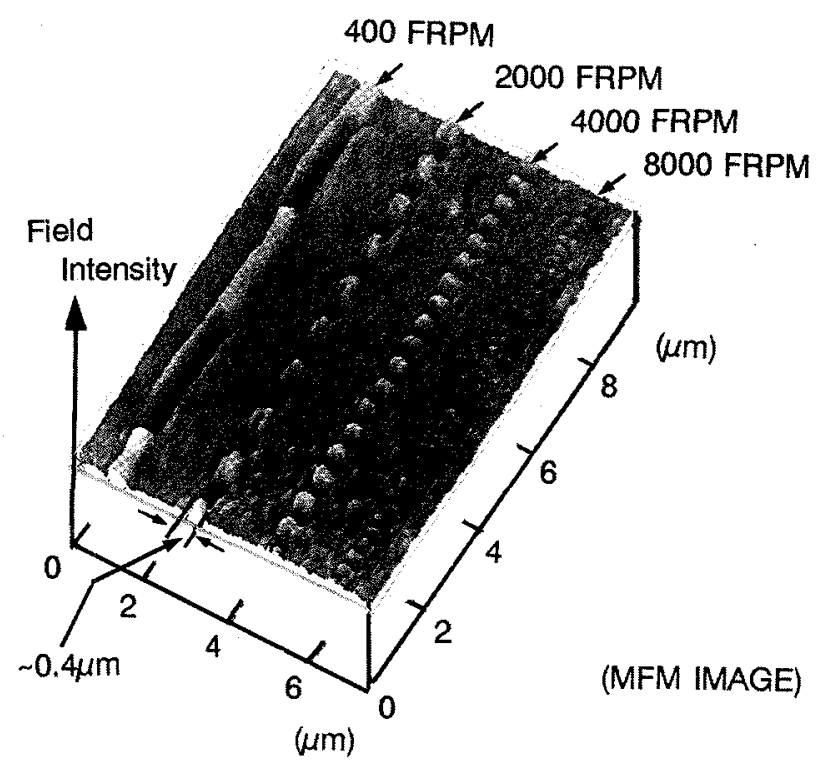

Fig. 11 Magnetic-force-microscopy observation of a perpendicular magnetic medium written by MPTW: Extremely high-density signal of 8000 FRPM can be observed even for $0.4 \mu \mathrm{m}$ track width.

\subsection{Signal processing for DWCR}

One of the signal processing candidates easily applicable to DWCR may be a multiple-valued signal processing with a multiple-track head. We suppose that a multiple-track single-pole head is composed of four narrow track subheads, whose track widths are set to be a ratio of $8: 4: 2: 1$ (Fig. 8). When each subhead magnetizes a magnetic storage medium to positive or negative saturation in correspondence to data 1 or 0,16 -valued readings can be realized with a single-track wide head.

For example, when '0000' (which is a part of a serial binary data row) is written in parallel by four narrow track subheads, the total magnetic flux detected collectively by a single-track wide head becomes the negative value of 15 
times that which is detected only from the track written by the narrowest subhead because all the tracks are magnetized in parallel to the negative saturation by the subheads (Table 1). When ' 1111 ' is written, the positive flux value of 15 times that of the narrowest subhead is detected because all the tracks are magnetized to the negative saturation. These flux values can be represented by the relative values of -15 and +15 which are normalized by the flux values detected from the narrowest track alone.

In the same manner, if '0001' (or '1110') is written, the relative flux value collectively read is -13 (or +13 ) because only the narrowest subhead magnetizes to the positive or negative saturation while the other three subheads magnetize to the opposite polarity. Furthermore, '0111' (or '1000') are read as the relative values of -1 (or +1 ) because only the widest subhead magnetizes to negative or positive.

Consequently, every serial 4 bits sliced from a serial data row can be converted through writing and reading into 16-

Table I Data conversion between serial 4 bit data row and 16-valued reading flux in DWCR with a multiple 4 track writing head and a single-track reading head.

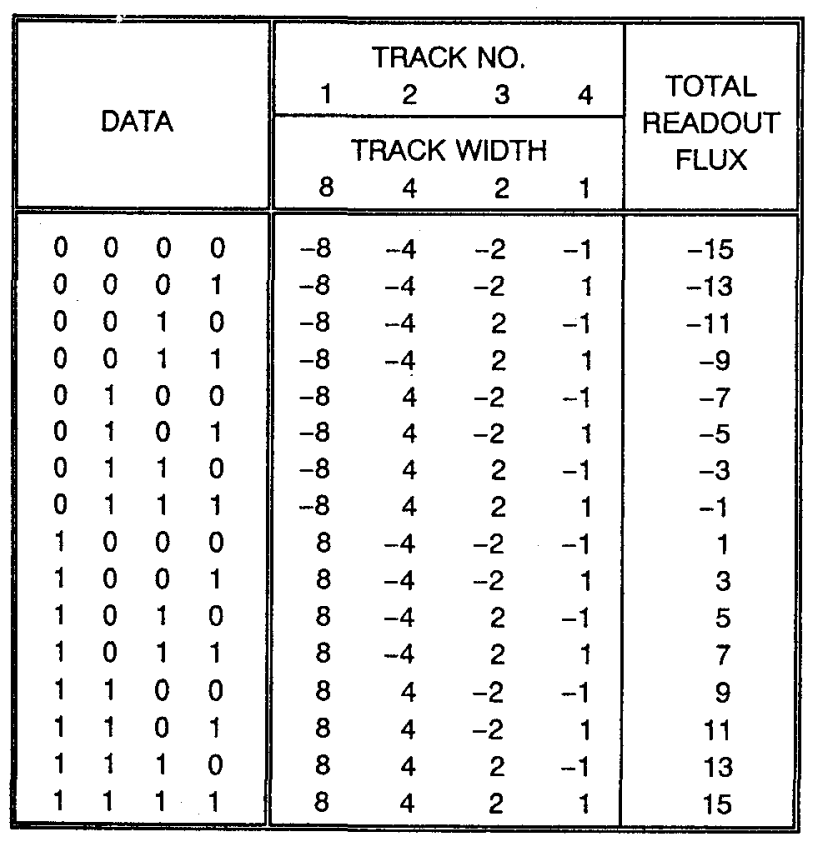

valued relative flux readings represented by odd numbers from -15 through +15 . The original serial digital data row can be reconverted from 16-valued readout voltages according to Table 1 .

When the track widths are set to $0.4,0.2,0.1$ and $0.05 \mu \mathrm{m}$ for each subhead and the guardbands to $0.3 \mu \mathrm{m}$, the total track width for four tracks of the multiple-track head becomes $1.65 \mu \mathrm{m}$. Therefore an areal bit density of over 1 $\mathrm{Gb} / \mathrm{cm}^{2}$, or about $10 \mathrm{~Gb} /$ inch $^{2}$ will be accomplished at a linear bit density of around 8 to $10 \mathrm{KFRPM}$. If more effective signal processing for DWCR and more precise two-dimensional microprocessing for making a multipletrack head can be developed, areal bit density will be increased significantly and will approached that of PSS.

\section{Conclusions}

Magnetic recording media essentially have the potential of an extremely high density exceeding $1 \mathrm{~Tb} / \mathrm{inch}^{2}$ at which a bit area corresponds to the size of a ferromagnetic singledomain fine particle or a parallel spin cluster. However we do not yet have a technique by which we can write each bit in each magnetic particle or each spin cluster and read them out. Although we are now writing and reading out data in several ten thousands of particles, the readable number of particles is decreasing year by year (from several thousands to several hundreds or tens) with increasing reading head sensitivity. In other words, magnetic recording is approaching spinic data storage.

To realize real spinic storage, a novel manufacturing method should be developed for spinic storage media and novel signal processing should be introduced for DWCR. A spinic storage head like a combination of a multiple track writing head and a single track inductive or magnetoresistive reading head should be developed to facilitate DWCR signal processing.

The author wishes to thank the members of his group, 
especially Dr. H. Muraoka, Dr. I. Tagawa, Dr. K. Takano, Mr. I. Watanabe and Mr. Y. Shimizu, for their valuable and helpful discussions, experiments and computer simulations.

\section{References}

[1] B. D. Cullity : "Introduction to magnetic materials", Addison-Wesley Publishing Company, 383-441(1972).

[2] S. Iwasaki and Y. Nakamura : "An analysis for the magnetization mode for high density magnetic recording", IEEE Trans. Magn., MAG-13, 1272(1977).

[3] I. Tagawa and Y. Nakamura : "Magnetic recording simulation and its applications", JMMM, 134, 382 (1994).

[4] Y. Nakamura : "Theoretical and experimental prospects of perpendicular magnetic recording", Jour. Mag. Soc. Jpn., 15, S2, 97(1991).

[5] I. Tagawa and Y. Nakamura : "Relationship between high density recording performance and particle coercivity distribution", IEEE Trans. Magn., 27, 4875(1991).

[6] Y. Nakamura : "Conditions for ultra-high density magnetic recording", Jour. Mag. Soc. Jpn., 13, S1, 33(1989).

[7] K. Ouchi : "Perpendicular recording media", Perpendicular magnetic recording' edited by S. Iwasaki and J. Hokkyo, Chap.7, Ohmsha, Tokyo, 109(1991).

[8] E. Koester : "Trends in magnetic recording media", JMMM, 120, 1(1993).

[9] N. R. Belk, P. K. George and G. S. Mowry : "Noise in high performance thin-film longitudinal magnetic recording media", IEEE Trans. Magn., MAG-21, 1350(1995).

[10] T. Yogi, T. A. Nguyen : "Ultra high density media : Gigabit and beyond", IEEE Trans. Magn., 29, 307 (1993).

[11] M. Futamoto, et al : "Investigation of $2 \mathrm{~Gb} / \mathrm{in}^{2}$ magnetic recording at a track density of $17 \mathrm{kTPI}$ ", IEEE Trans. Magn., 27, 5289(1990).

[12] E. S. Murdock, R. F. Simmons, R. Davidson : "Roadmap for 10 Gbit/in ${ }^{2}$ Media: Challenges", IEEE Trans. Magn., 28, 3078(1992).

[13] S. Yamamoto, Y. Nakamura, S. Iwasaki : "Extremely high bit density recording with single-pole perpendicular head", IEEE Trans. Magn., MAG-23, 2070 (1987).
[14] Y. Nakamura and I. Tagawa : "Possibilities of perpendicular magnetic recording", IEEE Trans. Magn., 24, 2329(1988).

[15] H. Muraoka and Y. Nakamura : "Multi-track submicronwidth recording with a novel integrated single pole head in perpendicular magnetic recording", IEEE Trans. Magn., 30, to be published, (1994).

[16] Y. Nakamura, H. Muraoka, I. Watanabe and T. Inaguma : "Submicron trackwidth recording utilizing a novel single pole head", Proc. PMRC94, 14pB-5, to be published, (1994). 